\title{
APPLICATION OF MACHINE LEARNING FOR ESTIMATING KENYAN MOTOR VEHICLE INSURANCE PREMIUM
}

\author{
Fidelia Pamba and Lucy Waruguru \\ KCA University \\ P.O. Box 56808 - 00200 Nairobi, Kenya
}

\begin{abstract}
Motor vehicle damage insurance is the most common type of insurance in the world and one that generates the largest amount of loss for most insurance companies. In Kenya especially, the challenge faced by insurers is to balance the growth of the motor vehicle insurance business by increasing the customer base while also maintaining the profitability of this sector. It is crucial to identify the main causes of motor vehicle damage, its impact on revenue for insurers and factors that contribute to high motor claims to enable more accurate estimates of risk versus premium paid. In recent years the interest has increased in the use of information technology (IT) and statistical machine learning methods, supported by increasing computing capabilities, data availability and the trend towards automation. Statistical regression models have numerous applications in this regard. This paper explores applicability of new machine learning techniques such as tree-boosted models to optimize the proposed premium of prospective policy holders. It proposes two machine learning models for pricing motor vehicle damage insurance (decision trees and regression). The aim is to identify sources of risks in motor vehicles and the variables for motor vehicle premium determination. Data from insurance companies has been used, which is made up of the premium rates and compensations, and other variables such as age, driver's experience, etc. Results will be used to advise the insurance companies on how to charge premiums dynamically.
\end{abstract}

\section{KEYWORDS}

Premium Estimation, Insurance, Machine Learning, Nairobi Kenya

\section{INTRODUCTION}

Motor vehicles in Kenya are the largest cause of accidents, damage and destruction of property (Peters et al., 2016). However, due to a low and uneven development of insurance in the developing society, companies rely on standardized measures for determining which premium to pay. The study carried out by IFAA in 2011 indicate, "out of the total 309,361 vehicles populated in Kenya only about 35\% or 106,765 were insured voluntarily whereas the remaining $65 \%$ were dependent on their financial resources if liability arose". Many insurers have been suffering losses after charging inadequate statutory premiums, problems in premium rate determination and monitoring, lack of integrated data and co-operation between the insurance industry, increased fraud and malpractice.

Although the insurance industry has grown rapidly in the industrialized countries, its growth in developing countries like Kenya has neither been satisfactory nor in tandem with the growth of other sectors of the economy. A key issue is how to price an insurance contract, which is also known as rate making. As the Insurance industry continues to grow in Kenya, research by the Association of Kenya Insurers (AKI) has shown that customers often pick an insurance company as purely based on the premium to be paid (AKI Annual Report, 2015). If the price is too high, customers will turn to other insurance companies, and if the price is too low, the insurance company will not receive enough premiums to cover the insureds' claim costs. 


\subsection{Background}

The insurance sector in East Africa is steadily developing, with its gross premiums totaling to $\$ 2.35 \mathrm{~b}$ across the region. Kenya's premiums form $75 \%$ of the total, and it leads the region with a $3 \%$ penetration (AKI annual report, 2015). However, insurance in the region is still underdeveloped as compared to the rest of the world.

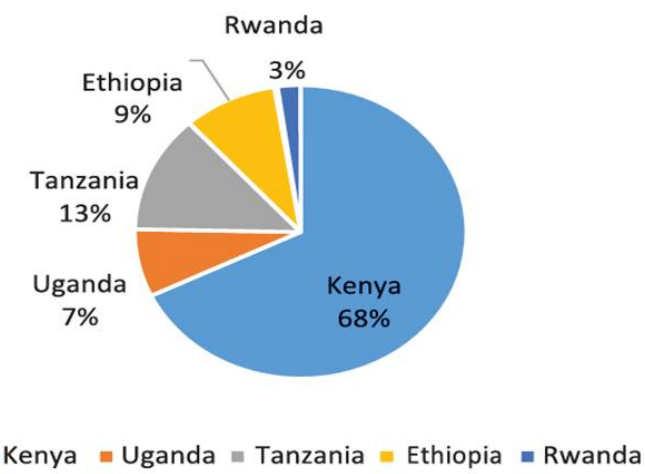

Figure 1. Gross written Premium in East Africa (From AKI Report 2015)

Although Kenyan insurance companies are the most well performing on almost the entire East Africa (See Figure 1), not all insurance products are generating high profits for the insurers. It has been discovered that motor vehicles generate losses every year because the amount of premium paid is very low compared to the cost of compensating damages from accidents. Transport plays a crucial role in facilitating socio-economic development.

\subsection{Problem Statement}

The high hazard of motor risk makes insurance companies to adopt various strategies to minimize motor insurance adverse effect and maximize their profitability. Different premiums or tariffs are applied depending on degree of risk that is highlighted within the insurance portfolio price (Mihaela, 2015). The main problem is that the level of premiums charged to a customer does not depend on statistical data about the state of road, age of vehicle, age and experience of the driver etc. (Dominique-Ferreira, 2017). The underwriting process involves investigation into various factors and analysing them through statisticians called actuaries. In many cases the actuaries are not accurate.

Recently, more sophisticated machine learning models such as logistic regression models and tree-based decision models have been developed, but their usage in the insurance industry is not widespread (Frees et al., 2014; Frees et al. 2016; Pozzolo, 2010) To the researchers' knowledge, a comparison of machine learning techniques to predict insurance premium at the local level is still lacking in the literature and will hence be explored here.

\section{MOTOR VEHICLE PREMIUM DETERMINATION}

As the Insurance industry continues to grow in Kenya, research by the Association of Kenya Insurers (AKI) has shown that customers often pick an insurance company as purely based on the premium to be paid (AKI Annual Report, 2015). This has made the need for accurate ratemaking more important than ever. The SAS Institute, (SAS, 2011) stated that multiple factors can be used to determine premium rates, and that as competition increases, insurers are introducing new and innovative rate structures. The critical question in ratemaking is, "What risk factors or variables are important for predicting the likelihood, frequency and severity of a loss?" Although there are many obvious risk factors that affect rates, subtle and nonnutritive relationships can exist among variables that are difficult, if not impossible, to identify without applying more sophisticated analyses. 
Traditional univariate analysis methods are outdated, and insurers have turned to multivariate statistical techniques such as generalized linear modelling to understand the relationships between multiple risk variables. Finally, insurers need to consider marketing costs, conversion rates and customer buying behavior in accurately pricing insurance products. It is becoming more evident that the key to gaining an edge in today's competitive market is the ability to quickly and efficiently explore, understand and act on the data to improve price competitiveness.

\subsection{Traditional Risk Estimation}

Common traditional methods include the centrally determined price option and the free market priced option as discussed below.

\subsubsection{Centrally Determined Price Option}

Most regulators have placed various restrictions on insurance policy coverage and rates, particularly for non-life motor vehicle insurance. Statutory tariffs are commonly imposed on compulsory insurance and strictly regulated by national legislation due to its sensitive nature. They prevent unsustainable price wars (Ernst \& Young, 2011). Unlike mature markets, prices are free and only subject to tough competition in most developing countries, compulsory premiums are statutory, and subject to government oversight, either directly or through some more complex governance process. In this case, the government is in charge of setting statutory in the form of setting of minimum and maximum or only maximum prices (Gönülal, 2010). The premium is calculated according to the risk + office expenses + other charges (Ben-Shahar \& Porat, 2016).

Different countries have pursued motor insurance tariff liberalization at different times and in different ways. In most countries statutory tariffs do not reflect claims costs and necessarily involve an element of cross subsidy and sometimes inhibit innovative pricing. As a result, this is an area where knowledge of the distinctive features is important and the trained of future market evolution will be a move towards partial de-tariff of MTPL insurance premium rating and full risk-based pricing typically correlates to market profitability and stability (Ernst \& Young, 2011).

\subsubsection{Free Market Priced Option}

In many developed and mature markets, prices are free and only subject to tough competition. In general, insurance risks can be very volatile, and the cost of meeting claims is constantly under pressure from inflation and other upward trends as a result the premium rating are very sensitive issue in insurance market. Most insurance policies are normally issued on a one-year renewable basis as a result in a competitive environment, an insurer can lose good business or gain bad business very quickly if its rates become out of line with the rest of the market. In this case, it is of vital importance for an insurer to keep rates under constant review and to amend them as necessary (Gönülal, 2010).

Competitive pricing will bring innovative product, new business idea, cheaper rates and variable discounts. However, the lower premiums force the insurer to be more selective to underwriting, and to accept only the better risks. It will also keep costs down and had economy administration and stricter claims procedures. This will have an impact on the general objectives of motor insurance (Einav et al., 2010).

\subsection{Insurance Prediction with Information Technology}

Different estimation techniques have been proposed in the past to be used to estimating the level of risk vs premium payment. From table 1 below, machine learning methods such as tree-based classification techniques are the best for predicting insurance premium. Several reasons explain their popularity: First, they are very easy to interpretable and communicate to a nontechnical audience. Second, they can handle both numeric and categorical predictors without any pre-processing. Third, they perform feature selection and can handle missing values explicitly, and any missing value is used as another level/numeric value (Kuhn and Johnson 2013). 
Table 1. Various methods for estimating risk level for insurance premium determination

\begin{tabular}{|c|c|c|c|}
\hline Technique & Methods & & \\
\hline & Econometric Model & Regression Model & Tree-based Classification \\
\hline Description & $\begin{array}{l}\text { A system of } \\
\text { interdependent } \\
\text { regression equations to } \\
\text { describe economic sales } \\
\text { or profit activity. } \\
\text { Parameters of the } \\
\text { equations are determined } \\
\text { simultaneously. These } \\
\text { models predict quite } \\
\text { accurately but are } \\
\text { relatively expensive to } \\
\text { develop }\end{array}$ & $\begin{array}{l}\text { Relates risk to other economic, } \\
\text { competitive or internal } \\
\text { variables and estimates an } \\
\text { equation using least squares } \\
\text { method. Relationships are } \\
\text { analyzed statistically. They } \\
\text { predict quite accurately, } \\
\text { especially in short and medium } \\
\text { term }\end{array}$ & $\begin{array}{l}\text { Consist of tree-like nested } \\
\text { if-then statements for } \\
\text { predictors that partition } \\
\text { data. This approach generates a } \\
\text { structure of "nodes" and } \\
\text { terminal "leaves", within } \\
\text { which a model is used } \\
\text { to predict the outcome. They } \\
\text { predict very accurately, } \\
\text { especially in short and medium } \\
\text { term }\end{array}$ \\
\hline $\begin{array}{l}\text { Accuracy } \\
\text { Short term (0-3 } \\
\text { months) }\end{array}$ & Good to very good & Good to very good & Very good to excellent \\
\hline $\begin{array}{l}\text { Medium term }(3 \\
\text { months - } 2 \text { years) }\end{array}$ & Very good to excellent & Good to very good & Very good to excellent \\
\hline $\begin{array}{l}\text { Long term (above } 2 \\
\text { years) }\end{array}$ & Good & Good & Very good \\
\hline Data Required & $\begin{array}{l}\text { Monthly or quarterly } \\
\text { history to obtain } \\
\text { meaningful } \\
\text { relationships. To have } \\
\text { more observations than } \\
\text { the no of independent } \\
\text { variables }\end{array}$ & $\begin{array}{l}\text { Same as in econometric } \\
\text { models }\end{array}$ & $\begin{array}{l}\text { Same as in econometric } \\
\text { models }\end{array}$ \\
\hline References & Evans (1969) & Clelland et al. (1966) & Quinlan (2004) \\
\hline
\end{tabular}

\section{METHODOLOGY}

\subsection{Data Collection}

Data collection was carried out by physically moving from organization to organization. Pre-established salient points and semi-structured questionnaires were used to uncover deeper meanings through a qualitative research design method using a multiple case study. The criteria and methods utilized for respondents' selection and contact are as follows:

- Insurance company's branch offices, which has been in operation to underwrite motor insurance since September 2011 are selected proportionally.

- Insurance officers directly involved in motor policy underwriting and/or claims handling are randomly selected from proportionally chosen insurance companies' branch office.

- $\quad$ Anyone who buys motor insurance policy and met accidentally at proportionally selected insurance company's branch office counter during the month of November 2016.

\subsection{Study Variables}

The study's independent variables included driver's experience, age, motorvehicle attributes, age and previous claims. The dependent variable was the level of risk, i.e. the probability for compensation. 


\subsection{Analysis Strategy}

The data analysis method applied for this study comprises both quantitative and qualitative methods. Quantitative method was used with a view to properly address the research questions and qualitative analysis method was employed to analyzed response on open-ended questionnaires. The data collected through close-ended questionnaires were analyzed with descriptive statistics. The data are presented using tables and charts. The researcher also utilized various descriptive statistical techniques such as mean, percentage and frequency distribution tables. The Waikato Environment for Knowledge Analysis (Weka) was used to conduct decision trees classification. For modeling the regression analysis, SPSS version 16.0 software tool was used.

To determine the variation between premium payment and motor vehicle risk factors, a regression model was applied in the form:

$$
y=\beta_{0}+\beta_{1} x_{1}+\beta_{2} x_{2}+\beta_{3} x_{3}+\beta_{4} x_{4}+\beta_{5} x_{5}+\beta_{6} x_{6}+\beta_{7} x_{7}+e
$$

Where $\beta 0$ is the intercept of the regression, $\beta 1$ to $\beta 5$ are the slopes representing the expected y value, and e represents the errors that should be normally distributed with mean 0 and equal variance. The independent variables are Policyholder (or driver) Experience (x1), Age (x2), Education (x3), Previous History (x4), Vehicle Type (x5), Vehicle Age (x6) and Lease Condition (x7).

\section{RESULTS}

This this section we provide results from the statistical analysis and interpretation of the data. In addition to descriptive analysis, we also provide results of machine learning through decision trees regression modeling using the sampled data.

\subsection{Demographic Outlook}

Demographic findings were based on the age and gender of respondents who completed the survey, the number of years of experience in driving, the employment status and frequency of driving a motor vehicle.

The largest age groups of respondents were aged between 18 years and 30 years, making up more than half of the survey participants. 31 to 40 years had the second largest number of survey participants. These are also usually the age groups where most drivers belong. Only $3 \%$ of the survey participants were 51 years and above.

On years of driving experience, most of the participants had less than 1-year experience making up $43 \%$. Most of these people filled the questionnaire on their way to take or renew their insurance policy. The next largest group had up to 3 years' experience (22\%). This group is important because they can share solid experience about insurance premium payment and claiming. Drivers with 3-5 years' experienced were the smallest group, making up 9\%. This group is also important for experience.

Luxury and sports cars received the highest compensation, either because of their high value or the likelihood to crash during a race. Private saloon cars and the truck and utility vehicles were almost similar in trends but also received high compensation. Minivans had the lowest compensation rate, especially in 2016 when less than $2 \mathrm{M}$ Kenyan shillings was paid out to compensate them.

\subsection{Classification Results}

The next part of the analysis was to develop a predictive model to predict the probability of claim given some possible risk factors on the occurrence of claims from the insurance industry. One of the ways to model insurance risk is through classification with Decision Trees. According to Duncan (1979), an advantage of the Decision Tree is that it is easy to interpret. The Decision Tree also works even if there is nonlinear relationship between variables. Finally, it does not require linearity assumption and it is not sensitive to outliers. 
Figure 2 shows the output from the classification for policyholders. The significant nodes $(p \leq 0.05)$ were displayed with the root of the decision tree being divided by the most important variable being the compensation amount. The policyholder experience highly determines compensation amount. Policyholders who had over 3 years driving experience are less likely to receive compensation because of an accident where PolicyHolder.Experience > 3.1 Years (215, with 173 Yes and 43 No). Age is also a good determinant, but on the negative side of risk. Younger drivers with less than a diploma for education, are the most likely to require high compensation amount where PolicyHolder. Age $\leq 31$ Years (198, with 133 Yes and 65 No).

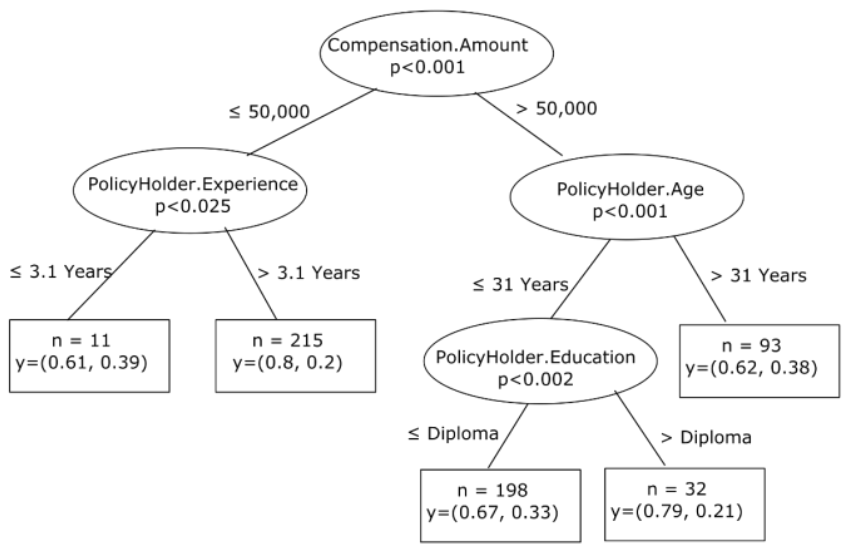

Figure 2. Classification output by Policyholder's characteristics

Figure 3 shows the output from the classification for motorvehicles. The significant nodes $(\mathrm{p} \leq 0.05)$ were displayed. The root of the decision tree is divided by the most important variable being the compensation amount. Motorvehicles with no previous accident history were less likely to need high compensation as a result of accidents where Previous. History $\leq 0$ (203, with 142 Yes and 61 No). Motorvehicles such as sports cars had the highest risk of getting accidents and receiving high amount of compensation, especially for vehicles driven for not more than 1 year where Vehicle.Type $\leq 2$ and Vehicle.Age $\leq 1$ Year (76, with 51 Yes and $25 \mathrm{No}$ ).

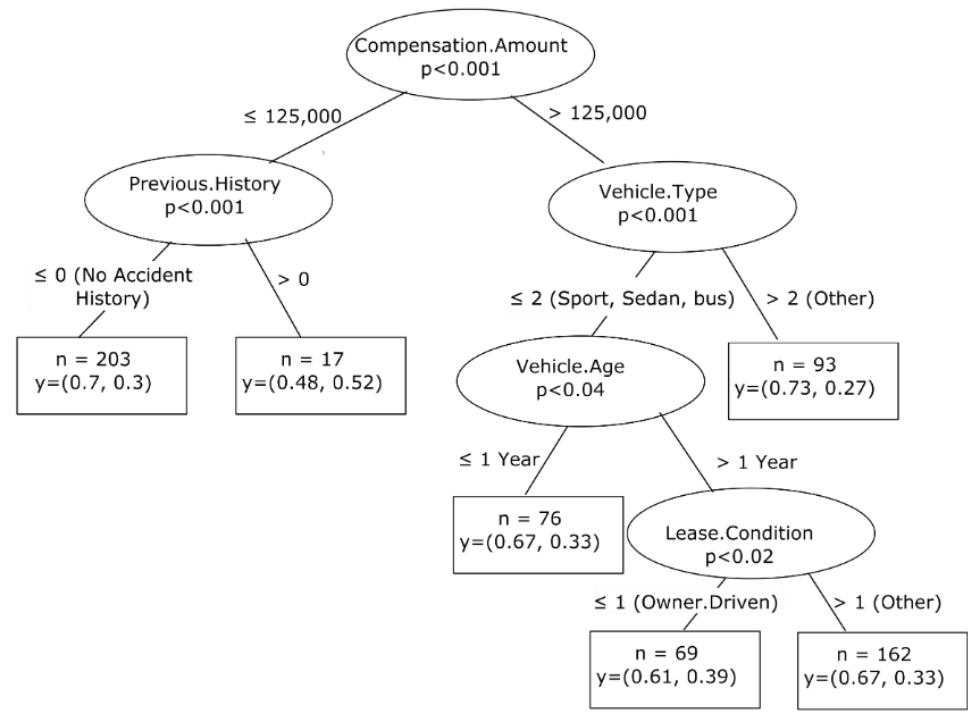

Figure 3. Classification output by Motorvehicle’s characteristics 


\subsection{Results from Regression}

Using results from the decision tree as guidance of which variables were good determinants for compensation, a regression model was created to test the dependent variable (Compensation) side by side with 7 independent variables: Policyholder Experience, Policyholder Age, Policyholder Education, Previous History, Vehicle Type, Vehicle Age and Lease Condition. Table 4.8 shows the estimation results. The previous history is the most significant variable with a t-statistic of 6.121 and p-value of 0.000 . Policyholder's (or driver's) experience was negative to the probability for claim, meaning that the longer the policyholder had driven the motor vehicle, the less he/she was likely to be involved in an accident. The same with Motor vehicle's age. It seems that older vehicles were less involved in an accident than newer vehicles. According to the regression results, all variables were significant except the policyholder's education level which was found not to be significant to the likelihood of being involved in an accident and claiming from the insurance company.

Table 2. Estimates from regression analysis of motorvehicle risk

\begin{tabular}{lllllcc}
\hline Variable & Coefficient & $\begin{array}{l}\text { Standard } \\
\text { Error }\end{array}$ & t-statistic & p-value & $\begin{array}{c}\text { 95\% confidence } \\
\text { interval }\end{array}$ \\
\hline P.EXP & -0.022 & 0.002 & 4.123 & $0.000^{* *}$ & 0.013 & 0.031 \\
P.AGE & 0.139 & 0.094 & 3.110 & $0.023^{*}$ & 0.060 & 0.218 \\
P.EDU & 0.307 & 0.105 & 1.977 & 0.061 & 0.139 & 0.581 \\
HIST & 0.358 & 0.198 & 6.121 & $0.000^{* * *}$ & 0.290 & 0.426 \\
V.TYPE & 0.128 & 0.057 & 2.844 & $0.021^{*}$ & -0.096 & 0.354 \\
V.AGE & -0.012 & 0.003 & 3.258 & $0.010^{* *}$ & -0.018 & -0.006 \\
L.COND & 0.417 & 0.126 & 3.315 & $0.002^{* *}$ & 0.398 & 0.421 \\
n & 260 & & & & & \\
$\mathrm{R}^{2}$ & 0.745 & & & & & \\
Adjusted $\mathrm{R}^{2}$ & 0.691 & & & & & \\
\hline
\end{tabular}

\section{CONCLUSION}

This paper has suggested a strategy for estimating motorvehicle insurance premium using statistical methods and information systems strategies. This section offers the theoretical and industrial implications of the study, with its limitations and propositions for future direction.

\subsection{Discussion}

The primary objective of this study was to establish machine learning models for regulating motor vehicle premiums among insurance companies. Results from this primary objective and associated specific objectives can help to investigate consequences of action by policyholders in a buyer-seller relationship in the car insurance market.

On average, policyholders pay KSh.3, 950 per month, about KSh.50, 000 per year. The highest standard deviation is on duration of vehicle ownership, which is almost the same as the mean indicating a lot of variation. These findings have been discovered in other studies such as Desalegn (2014) and Poposki et al. (2015). This shows that in general insuring a motor vehicle is an expensive investment. It is important for policyholders to get value for their money, and understanding how key variables influence their ability to receive compensation can help a lot. Other key findings were that the newly registered vehicles of 0-12 months had the highest number of compensation from theft or accident and that Luxury and sports cars received the highest compensation. These two findings are also similar with findings from other researchers (e.g. Müller and Te, 2017; Stevenson et al., 2017; Lesmana et al, 2018) and shows that drivers of such vehicles should be charged a much higher premium than any other groups.

All the results from the first three specific objectives were agreeing with each other, and the results from both decision trees and regression model were quite similar in identifying the most influencing variables. As based on the specific objective 4 which was to test the models, it can be reported that all the models were quite accurate. For example, analysis of covariance was done for age against the amount compensated in KSh. This was done separately for male and female participants but both genders showed the same pattern. 
People were more likely to need compensation between 25 and 35 years old. Younger people also required high compensation, but after 60 years the compensation rate was very low.

The general observation from the results of this study is that several factors are important for determining risk in addition to the motor vehicle insurance premium value.

\subsection{Limitations and Future Research Areas}

Although the results from this study are encouraging, further work is needed before the procedures are used in practice. First, analysis using a larger sample of data is necessary as the study has used a limited sample. Secondly particular attention should be made to how well the assumptions behind the credibility model are fulfilled and whether improved variables for the model can be found.

Finally, the results of using the procedures described here should also be compared with present practice, where estimates of risk premium from the identified variables are, in effect, subjectively combined. It is expected that a dramatic improvement will be discovered, and that the proposed procedures described in this thesis will enable the process of motor vehicle insurance classification to be put on a more objective footing.

\section{REFERENCES}

AKI Annual Report 2015. Association of Kenya Insurers Annual Report, 2015

Ben-Shahar, O., \& Logue, K. D. 2016. The perverse effects of subsidized weather insurance. Stan. L. Rev., 68, 571.

Dal Pozzolo, A. 2010. Comparison of Data Mining Techniques for Insurance Claim Prediction. SAS 2011. SAS Institute Inc., Cary, NC, USA.

Desalegn, A. 2014. Assessment of Motor Insurance Business on Financial Performance of Insurance Company, The Case of Awash Insurance Company. PhD diss., St. Mary's University.

Dominique-Ferreira, S. 2017. How important is the strategic order of product attribute presentation in the non-life insurance market?. Journal of Retailing and Consumer Services, 34, 138-144.

Duncan, R. 1979. What is the right organization structure? Decision tree analysis provides the answer. Organizational Dynamics, 7(3), 59-80.

Einav, L., Finkelstein, A., \& Levin, J. 2010. Beyond testing: Empirical models of insurance markets. Annu. Rev. Econ., 2(1), 311-336.

Evans, M. K. 1969. Macroeconomic activity: Theory, forecasting, and control; an econometric approach. Harper \& Row.

Farrell, D., Wheat, C., \& Mac, C. 2017. Paying a Premium: Dynamics of the Small Business Owner Health Insurance Market.

Frees, E.W., G. Meyers, and R.A. Derrig. 2016. Predictive Modeling Applications in Actuarial Science: Volume 2, Case Studies in Insurance. International Series on Actuarial Science. Cambridge University Press.

Gönülal, S. 2010. Motor third-party liability insurance.

Kuhn, M., and K. Johnson. 2013. Applied Predictive Modeling. Springer

Lesmana, E., Wulandari, R., Napitupulu, H., \& Supian, S. 2018. Model estimation of claim risk and premium for motor vehicle insurance by using Bayesian method. In IOP Conference Series: Materials Science and Engineering Vol. 300, No. 1, p. 012027. IOP Publishing

Mihael, D. 2015. A review of theoretical concepts and empirical literature of non-life insurance pricing. Procedia Economics and Finance, 20, 157-162.

Müller, D., \& Te, Y. F. 2017, December. Insurance premium optimization using motor insurance policies-A business growth classification approach. In Big Data (Big Data), 2017 IEEE International Conference on (pp. 4154-4158). IEEE.

Peters, J. A. J., Ferguson, D., Madigan, R., \& McKenna, T. 2016. U.S. Patent Application No. 14/607,433.

Stevenson, M., Harris, A., Mortimer, D., Wijnands, J. S., Tapp, A., Peppard, F., \& Buckis, S. 2017. The effects of feedback and incentive-based insurance on driving behaviours: study approach and protocols. Injury prevention, injuryprev-2016.

Temesgen, Z. B. 2004. Determinants for Effective Application of Software in Computer Assisted Auditing Techniques (CAATs).

Yang, Y., Qian, W., \& Zou, H. 2018. Insurance premium prediction via gradient tree-boosted Tweedie compound Poisson models. Journal of Business \& Economic Statistics, 36(3), 456-470. 